\title{
Satisfacción de los estudiantes de una facultad de medicina peruana
}

\author{
Satisfaction of medical students of a Peruvian school of medicine \\ Javier Cieza Zevallos ${ }^{1, a}$, Alessandra Bertha Castillo Velásquez ${ }^{1, b}$, Francis Astrid Garay Buitrón ${ }^{1, b}$, \\ Jonnathan Jesús Poma Gálvez ${ }^{1, b}$
}

\section{RESUMEN}

Objetivos: Evaluar la satisfacción de los estudiantes de una facultad de medicina peruana en el año 2014 Material y métodos: Estudio descriptivo, observacional y transversal. Se aplicó la encuesta SERVQUAL a una muestra de los alumnos que cursaron el primer semestre del año 2014 en una facultad de medicina peruana. Se calculó la satisfacción restando el promedio de las preguntas de percepción con el puntaje de la expectativa. Se consideró satisfacción a una diferencia mayor o igual a 0. Se usó el paquete estadístico SPSS 18.0 y se analizaron las variables con el modelo de regresión logística multinominal y la prueba de chi cuadrado. Se consideró el valor p $<0,05$ como estadísticamente significativo. Resultados: De 188 encuestados, 54,8\% eran varones. La mayoría provenía del área de Lima Centro (42\%), Santiago de Surco y Miraflores (20\%). El 100\% de los encuestados era soltero y $14,9 \%$ eran beneficiarios de subvención económica. El porcentaje de satisfechos fue de 8\%; el de insatisfacción leve, 66\%; el de moderada, 23\% y 3\% estuvo muy insatisfecho. Solo se encontró relación estadística entre la edad y los elementos tangibles. Conclusiones: La magnitud de estudiantes satisfechos más grados leves de insatisfacción fue de $73,4 \%$. Las dimensiones con mayor y menor satisfacción fueron la de elementos tangibles y capacidad de respuesta, respectivamente. No parece haber relación significativa con las variables de las personas evaluadas, salvo con la edad en algunas dimensiones.

PALABRAS CLAVE: Satisfacción personal, estudiantes de medicina, control de calidad, educación superior, educación médica, facultad de medicina. (Fuente: DeCS BIREME).

\section{SUMMARY}

Objectives: To determine the satisfaction of medical students of a Peruvian school of medicine in 2014. Methods: Cross-sectional study carried-out among medical students of the first semester of 2014 in whom a SEVQUAL survey was applied. Satisfaction was calculated by subtracting expectation minus perception, with satisfaction defined to a difference above 0 . SPSS 18.0 was used with chi square and logistic multinomial regression tests. A p value $<0.05$ was considered statistically significant. Results: A total of 188 students were surveyed; 54.8\% were males; 42\% come from Lima Centro; Santiago de Surco and Miraflores (20\%); all were ingle and 14\% received scholarships. Percentage of satisfaction was $8 \%$; mild no satisfaction was seen in $66 \%$, moderate no satisfaction in $23 \%$ and $3 \%$ were no satisfied. Only age and tangible elements reached statistical significance. Conclusions: The proportion of satisfied and mild no satisfaction reached $73.4 \%$. Higher no satisfaction was observed with tangible elements and lower no satisfaction with capacity of response.

KEYWORDS: Personal satisfaction, students, medical, quality control, education, higher, schools. (Source: MeSH NLM).

\footnotetext{
Facultad de Medicina Alberto Hurtado, Universidad Peruana Cayetano Heredia. Lima, Perú.

Doctor en Medicina;

Estudiante de Medicina
} 


\section{INTRODUCCIÓN}

La calidad se define como el conjunto de propiedades que permiten juzgar el valor de un elemento $(1,2)$; en el caso de un servicio brindado se tiene en consideración el proceso en conjunto (3-7).

Una manera de evaluar la calidad es a través de la satisfacción y existen diversos instrumentos para su medición, uno de ellos es la encuesta SERVQUAL. Esta define satisfacción como la brecha entre lo que uno espera recibir (expectativa) y la experiencia percibida luego de recibir el servicio (percepción) $(8,9)$.

Este instrumento integra las características de un servicio en 5 dimensiones generales (4-6): a) Elementos tangibles: La imagen del proveedor del servicio y a los elementos en contacto con el cliente, desde infraestructura, equipamiento hasta recursos humanos; b) Fiabilidad: La capacidad del proveedor para cumplir de manera eficaz con el servicio que ofrece; c) Capacidad de respuesta: Disposición para satisfacer las necesidades de los clientes en el menor tiempo posible; d) Seguridad: Habilidad de los empleados para inspirar confianza al cliente sobre el servicio que está recibiendo, y; e) Empatía: Identificación del prestador con las necesidades del cliente, "alinear" las ideas del cliente con las de la institución.

La encuesta SERVQUAL ha sido aplicada mundialmente y en diversas actividades. En Irán, en 2008 , se encontró que los alumnos de una universidad de ciencias médicas tenían bajos niveles de satisfacción en todas las dimensiones evaluadas por la encuesta SERVQUAL. Estos resultados fueron similares a los reportados por otros investigadores de la zona $(5,9)$.

En nuestro país, una investigación en una facultad privada de Medicina en el 2002, encontró que la confiabilidad fue la dimensión con menor satisfacción de todas; los autores resaltaban que la dimensión de aspectos tangibles fue la que presentó menor expectativa frente a las otras (10). En el 2008, se evaluó la satisfacción de los estudiantes de medicina de dos universidades, una privada y otra estatal, se encontró que los alumnos tenían mayor satisfacción en la privada comparado con la estatal; asimismo, se encontró que el género, año de estudios, edad, ocupación de los padres, lugar de residencia y estado civil de los estudiantes influían en la satisfacción, en ambos establecimientos (11).
En el 2013, una universidad nacional evaluó la satisfacción de los alumnos del curso de Pediatría Comunitaria; se aplicó el instrumento antes y después de llevar la rotación, encontrándose que la mayoría de los alumnos estaban satisfechos (7).

Es importante conocer el estado actual y procurar brindar la mejor formación a estos profesionales (9). En este sentido, no hay estudios recientes sobre la calidad del servicio brindado.

El objetivo del estudio fue evaluar la satisfacción de los estudiantes de una facultad peruana de medicina usando la encuesta SERVQUAL. De manera secundaria determinar las dimensiones con mayor y menor satisfacción, si existen cambios en el grado de satisfacción al transcurrir la carrera y si existen diferencias en la satisfacción en función de la edad, sexo, estado civil, estado socio-económico.

\section{MATERIAL Y MÉTODOS}

Estudio descriptivo en 895 estudiantes de la carrera de Medicina de una universidad privada, matriculados en el segundo semestre del 2014. El tamaño de la muestra se determinó usando un intervalo de confianza del $95 \%$, potencia de $80 \%$ y un margen de error del $10 \%$. El tamaño de la muestra calculado fue de 188 alumnos, se previno una falta de colaboración del $10 \%$ por lo que la muestra final calculada fue 207 personas. La recolección de datos se realizó desde fines de 2014 hasta mediados de 2015.

Se utilizó una variación en la encuesta original SERVQUAL y se adaptó a los servicios ofrecidos por la facultad de medicina tanto a nivel académico como a nivel administrativo. Se respetaron las cinco dimensiones evaluadas originalmente (elementos tangibles, fiabilidad, capacidad de respuesta, seguridad y empatía) y el número de preguntas para evaluar las expectativas fueron $5 \mathrm{y}$ para las percepciones, 22. Se decidió usar solo 5 preguntas en la parte de expectativas debido a que el estudio previo que se realizó en esta misma facultad por Cieza et al (10), en el 2002, utilizó esta variación.

Se trabajó con una versión en físico y una virtual dependiendo de la preferencia y disposición del encuestado. En ambos casos, la encuesta fue anónima y aplicada por el mismo entrevistado. Adicionalmente a la encuesta, tanto en forma virtual como física, se hizo llegar información sobre el trabajo al participante conforme con lo establecido por el Comité de Ética de la Universidad Peruana Cayetano Heredia. 
La encuesta se dividió en dos partes: Parte A con cinco preguntas para evaluar expectativas y la parte B con 22 preguntas, para evaluar la percepción (experiencia). Se utilizó la escala de Likert, en la parte A, 1 equivalía a lo menos importante para la persona y 5 lo más importante, y en la parte $\mathrm{B}, 1$ equivalía a estar en total desacuerdo con lo que afirmaba el ítem y 5 totalmente de acuerdo.

Se registraron los siguientes datos referentes a cada estudiante: sexo, edad, estado civil, distrito de residencia, si recibe o no subvención económica y el año de estudio en el 2014. Para el análisis, la variable de edad se estratificó en 4 grupos: 18 a 19, 20 a 21, 22 a 23 y mayor a 24 años; los distritos de residencia se agruparon en Lima norte, Lima centro, Lima este, Callao-Sur y Santiago de Surco-Miraflores.

Para evaluar la satisfacción global de los estudiantes, se obtuvo el promedio simple de todas las preguntas de la parte B ("Percepción") y se restó con el promedio simple de todas las preguntas de la parte A ("Expectativa"). Se realizó el mismo proceso para calcular la satisfacción para cada una de las cinco dimensiones, según las preguntas correspondientes (Tabla 1).

Para ambos cálculos una diferencia de cero o algún número positivo se registró como satisfacción y una diferencia de algún número negativo se registró como insatisfacción. La insatisfacción se estratificó como: muy insatisfecho (puntaje de $-4,00$ a $-2,51$ ), insatisfacción moderada (puntaje de $-2,50$ a $-1,51$ ) e insatisfacción leve (puntaje de -1,50 a -0,01).

la variable en estudio fue la satisfacción y fue definida como satisfacción o insatisfacción, mientras que las variables independientes fueron: sexo, edad, estado civil, distrito de residencia, subvención económica y el año de estudios. El análisis se realizó con el paquete estadístico SPSS v 18 y se aplicó la prueba de regresión logística multinominal para

Tabla 1. Preguntas correspondientes a cada dimensión.

\begin{tabular}{lcc}
\hline DIMENSIÓN & PARTE A & PARTE B \\
\hline Elementos tangibles & E1 & P1-P4 \\
Fiabilidad & E2 & P5-P9 \\
Capacidad de respuesta & E3 & P10-P13 \\
Seguridad & E4 & P14-P17 \\
Empatía & E5 & P18-P22 \\
\hline
\end{tabular}

determinar asociación entre las variables siguiendo el modelo paso a paso retirando progresivamente una a una las variables de confusión; luego de ello se usó la prueba del chi cuadrado. Se consideró estadísticamente significativo un $\mathrm{p}<0,05$.

\section{RESULTADOS}

De los 207 participantes, 192 llenaron la encuesta voluntariamente, de las cuales 188 encuestas cumplieron a plenitud las exigencias del estudio y fueron incluidas en el estudio. El 54,8\% fue del sexo masculino; la mayor parte de estudiantes provinieron de distritos considerados como Lima Centro, y el 20\% de los distritos de Santiago de Surco y Miraflores. Todos los encuestados eran solteros y $14,9 \%$ tenían subvención económica (Tabla 2).

Tabla 2. Características de la muestra de estudio.

\begin{tabular}{lrr}
\hline CARACTERISTICA & n & \% \\
\hline Sexo & & \\
Femenino & 85 & 45,2 \\
Masculino & 103 & 54,8 \\
Año de estudio & & \\
$1^{\circ}$ & 32 & 17,0 \\
$2^{\circ}$ & 38 & 20,2 \\
$3^{\circ}$ & 33 & 17,6 \\
$4^{\circ}$ & 26 & 13,8 \\
$5^{\circ}$ & 25 & 13,3 \\
$6^{\circ}$ & 14 & 7,4 \\
$7^{\circ}$ & 20 & 10,6 \\
Procedencia & & \\
Norte & 28 & 14,9 \\
Lima Centro & 79 & 42,0 \\
Este & 34 & 18,1 \\
Callao-Sur & 9 & 4,8 \\
Surco-Miraflores & 38 & 20,1 \\
Subvención económica & & \\
Sí & 28 & 14,9 \\
No & 160 & 85,1 \\
Edad & & \\
18-19 años & 47 & 25,0 \\
20-21 años & 54 & 28,7 \\
$22-23$ años & 46 & 24,5 \\
24 a más & 41 & 21,8 \\
\hline & &
\end{tabular}


Satisfacción e insatisfacción global y según dimensión

Del total de encuestados, el $8 \%$ manifestó estar satisfecho, $66 \%$ con insatisfacción leve, $23 \%$ moderada y $3 \%$ muy insatisfecho.

La dimensión que presentó mayor expectativa fue la de fiabilidad con promedio de 4,65 y la de menor expectativa fue la de elementos tangibles con 4,0.

El mayor porcentaje de estudiantes satisfechos $(27,6 \%)$ se encontró en la dimensión de elementos tangibles y el menor, en capacidad de respuesta $(9,04 \%)$ (Gráfico 1).

\section{Satisfacción según año de estudio}

La satisfacción e insatisfacción fue similar a lo largo de la carrera y la mayor proporción correspondió a un grado de insatisfacción leve. En los años de prácticas pre-profesionales (sexto y sétimo año) y en el cuarto año no hubo muy insatisfechos (Gráfico 2).

\section{Relación de dimensiones con variables independientes}

En la dimensión de elementos tangibles, el análisis univariado mostró asociación estadística entre edad y satisfacción ( $\mathrm{p}=0,02)$; el análisis de regresión logística multinominal no mostró asociación significativa cuando se incluyeron todas las variables independientes en general $(p=0,10)$, aunque la variable edad sí mostró significancia estadística $(p=0,02)$. Al eliminar el área de procedencia y sexo, pues se consideraron variables de confusión, se encontró significancia estadística $(\mathrm{p}=0,03)$, permaneciendo la edad como la única variable significativa dentro del modelo.

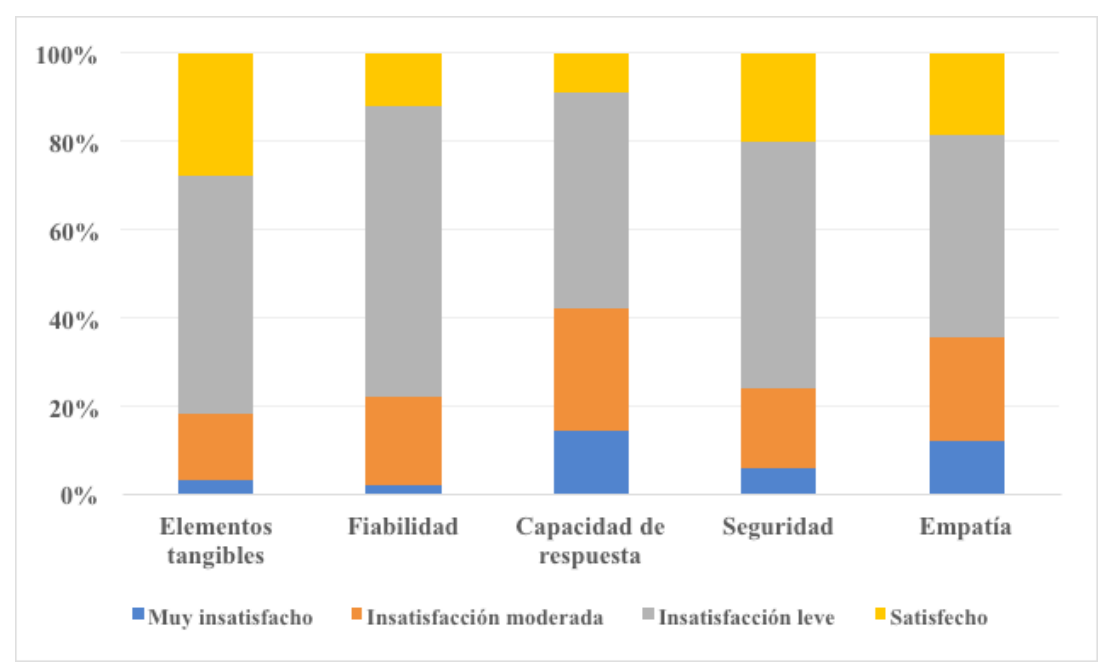

Gráfico 1. Satisfacción e insatisfacción según dimensiones.

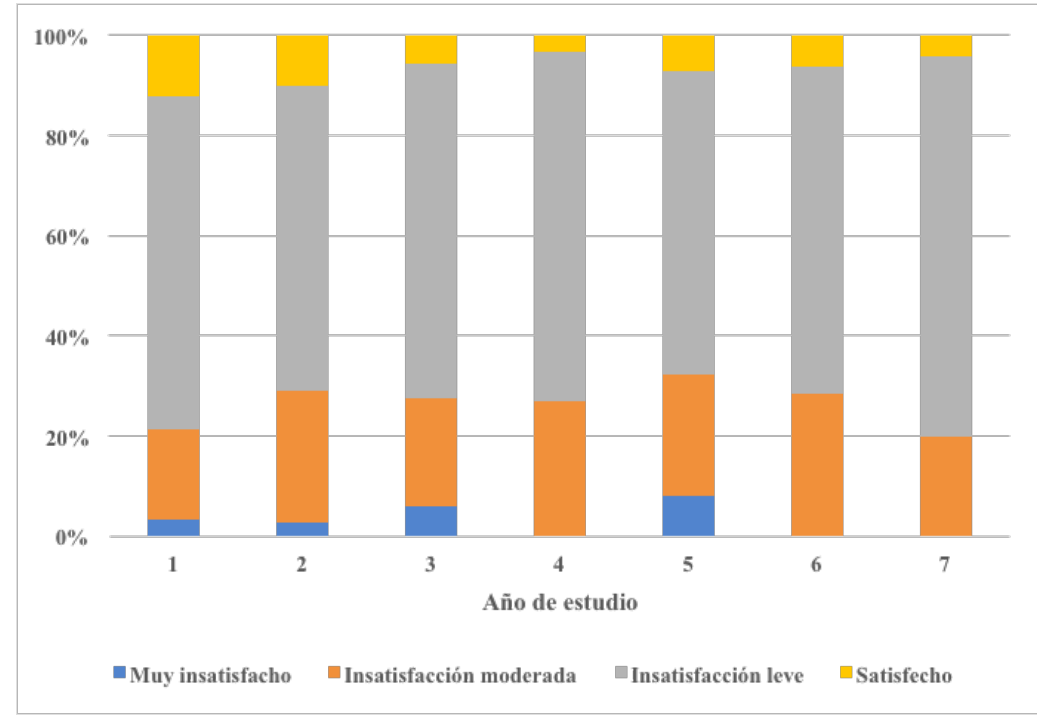

Gráfico 2. Satisfacción e insatisfacción según año de estudio. 
No se encontró relación significativa con las variables independientes en general ni en el análisis univariado en la dimensión fiabilidad $(\mathrm{p}=0,71)$, ni en la dimensión empatía $(p=0,42)$.

En la dimensión capacidad de respuesta, el análisis de regresión logística multinominal incluyendo todas las variables independientes no mostró significancia estadística $(\mathrm{p}=0,10)$, aunque se encontró asociación significativa en la variable edad $(\mathrm{p}=0,02)$. Sin embargo, en el análisis univariado, la edad no mostró significancia estadística $(\mathrm{p}=0,13)$.

En la dimensión seguridad, en el análisis de regresión logística multinominal incluyendo todas las variables independientes no se encontró significancia estadística $(\mathrm{p}=0,16)$; pero, la variable año de estudio mostró asociación significativa $(\mathrm{p}=0,03)$. Sin embargo, en el análisis univariado el año de estudio no mostró significancia estadística $(\mathrm{p}=0,06)$.

No se encontró relación significativa de la satisfacción global con las variables independientes ni en conjunto $(\mathrm{p}=0,73)$, en la regresión logística multinominal, ni en el análisis univariado.

\section{DISCUSIÓN}

Debido a que la recolección de datos se realizó desde fines de 2014 hasta el 2015, alumnos de la misma promoción fueron encuestados en diferentes semestres $o$ en diferentes circunstancias lo que podría haber influenciado en la satisfacción que el mismo alumno hubiera tenido de ser encuestado en otro momento, por ello se consideró el año que se cursó en el 2014 para asegurar que por lo menos todos habían pasado por experiencias similares. Por otro lado, la naturaleza del estudio no permite inferir causalidad; sin embargo, se pueden observar algunas relaciones, por lo que harían falta más estudios para evaluar asociación entre la satisfacción y otras variables, de manera que se pueda hacer una intervención más enfocada en actuar sobre las debilidades y mejorar las fortalezas de la institución.

Al recolectar las encuestas, los participantes mencionaron que no se debería combinar la opinión sobre el tema administrativo con el tema educativo pues una percepción baja sobre alguna de las áreas podría afectar la satisfacción general. Esto se debería tomar en cuenta en futuros estudios para tener una idea más clara sobre las áreas a trabajar; sin embargo, este estudio es una buena aproximación para conocer la opinión de los usuarios.
Según el análisis, la dimensión que presentó mayor expectativa fue la de fiabilidad, es decir que los estudiantes consideran muy importante que el servicio, sin especificar administrativo o docente, se cumpla en el plazo prometido. Por otro lado, la dimensión que presentó menor expectativa fue la de elementos tangibles que se refiere tanto a la apariencia de los ambientes como a la del personal que los atiende.

Los rangos para considerar satisfacción e insatisfacción se han estructurado en el concepto de cuartiles de los resultados. Al respecto, es importante subrayar que la diferencia entre satisfechos e insatisfechos leves ha sido muy pequeña por lo que la interpretación de los resultados debería tomar en consideración este concepto, más aún, considerando que la satisfacción es una variable cualitativa y al tratar de medirla uno puede establecer diferentes puntos de corte pues no hay un consenso establecido. La satisfacción global fue $8 \%$, los levemente insatisfechos fueron la mayoría de la población estudiada $(65,4 \%)$ y su puntaje fluctuó entre $-1,50$ y -0,01. La diferencia entre satisfechos y levemente insatisfechos es mínima ya que la persona que mostró la mayor satisfacción lo hizo con un puntaje de 0,91 . Esto es positivo ya que no existe una amplia brecha entre ambos grupos por lo que lograr un mayor porcentaje de satisfacción es totalmente plausible.

Las variables independientes (sexo, edad, área de procedencia, etc), no parecen tener una relación clara con la satisfacción global, esto difiere con lo encontrado en el estudio realizado en una universidad privada y otra nacional en el que sí se encontró relación estadísticamente significativa (10). En nuestro estudio, se vio que no era solo una variable la que influenciaba en la satisfacción, sino era la combinación de dos o más. Es probable, que en la satisfacción también intervenga la manera en que una persona procesa la información que recibe y esto a su vez es influenciado por muchos factores que no han sido evaluados en este estudio.

En el análisis por cada dimensión, solo hubo asociación estadísticamente significativa con la edad en la dimensión de elementos tangibles. Sin embargo, se observó que la edad también parece influir en la satisfacción en la dimensión capacidad de respuesta, mas no lo hace como variable única. Es pertinente indicar que la variable edad no es en sí modificable, pero sí se podría indagar de manera más específica cuáles son las expectativas de los alumnos para ofrecer un mejor servicio y esto solo es posible con una investigación de tipo cualitativa. 
Si bien es cierto que la edad podría estar relacionada a la madurez de la persona, no siempre puede existir esta correspondencia y esto estaría reflejando las divergencias de relaciones de la edad, el año de estudio y ciertas variables de satisfacción como elementos tangibles, seguridad y fiabilidad.

La dimensión que presentó mayor satisfacción fue la de elementos tangibles y como se mencionó, estaba relacionada con la edad de los estudiantes. Cuando se evaluaron las preguntas de percepción, se observó que el mayor puntaje fue otorgado a la apariencia del personal y el menor al diseño y a lo visualmente atractivo que son los establecimientos donde se brinda la atención. Es pertinente mencionar que durante la época de aplicación de la encuesta se estaba realizando modificaciones estructurales en una de las sedes más relevantes de la Universidad.

La dimensión que presentó menor satisfacción fue la de capacidad de respuesta; esta dimensión no fue la que presentó la mayor expectativa y registró el mayor porcentaje de "muy insatisfechos". Los estudiantes percibieron que cuando hacían una solicitud no recibían una atención rápida; sin embargo, hay una tendencia a reconocer que el personal sí está dispuesto a ayudarlo. Se encontró que la edad era una variable importante para esta dimensión, pero no fue lo único que influía en su satisfacción. Si bien la relación no fue significativa con el área de procedencia, resalta que los habitantes del Sur y del Callao (periferia) presentan la menor satisfacción y a su vez el mayor porcentaje de muy insatisfechos. En este sentido, es conveniente evaluar el uso de medios que reduzcan el tiempo que invierte el alumno en su desplazamiento hacia la facultad.

Al analizar el año de estudio en las dimensiones y la satisfacción global, no se encontró relación significativa. Inicialmente parecía haber relación con la dimensión de seguridad, pero el análisis ulterior no mostró significancia estadística. En esta dimensión, se observó que los alumnos de cuarto y sétimo año fueron los que presentaron mayor insatisfacción. La pregunta con menor percepción se refiere a la seguridad con el servicio brindado. Esto podría interpretarse en función del cambio del modelo de aprendizaje que ocurre en cuarto año cuando se inicia la práctica clínica, y los profesores no son necesariamente homogéneos en su comunicación y además, la realidad de la práctica médica se contrasta con lo aprendido en los años de ciencias básicas. En el caso de los internos, se podría hipotetizar una variante cualitativa en su desarrollo académico que es el asumir responsabilidades prácticamente similares al del médico en ejercicio. Esta situación implica mayor intensidad en el trabajo, mayor responsabilidad y una situación emocional mucho más exigente. A su vez permite una evaluación mucho más profunda del médico con quien interactúa, que en general es el médico residente, quien aún está en formación.

En los estudiantes del 5to año se observó una mayor frecuencia de muy insatisfechos que desaparece en los años 6to y 7mo; esto puede tener muchas interpretaciones, desde situaciones coyunturales hasta otras que no se pueden responder mediante este estudio.

Es necesario entender que la satisfacción depende de las expectativas y éstas de la experiencia previa o vivencias personales sobre las cuales la facultad no tiene control, conocer las expectativas es importante para dirigir las mejoras en las áreas donde se requiera.

Asimismo, es importante evaluar las percepciones de manera que se puedan realizar las mejoras pertinentes y mantener lo bueno para que el nivel de satisfacción mejore. Es oportuno comentar, que todo este esfuerzo por mejorar el servicio debe buscar que el alumno se prepare en un ambiente positivo y con las herramientas adecuadas para que su desarrollo como futuro profesional alcance el óptimo posible.

En conclusión, la magnitud de estudiantes satisfechos más grados leves de insatisfacción fue de $73,4 \%$. Las dimensiones con mayor y menor satisfacción fueron la de elementos tangibles y capacidad de respuesta, respectivamente. No parece haber relación significativa con las variables de las persona evaluadas, salvo con la edad en algunas dimensiones.

\section{Contribución de autoría:}

ABCV, FAGB y JJPG: Redacción del protocolo, ejecución del estudio, procesamiento e interpretación de los datos, redacción del artículo. JCZ: Diseño del estudio y revisión crítica del manuscrito.

\section{Declaración de financiamiento y de conflictos de intereses:}

El estudio fue financiado por los investigadores. Los autores declaran no presentan conflictos de interés. 


\section{Correspondencia:}

Jonnathan J. Poma Galvez

Jonnathan.poma@upch.pe

Av. Brasil 861 Dpto 1406 A

Jesús María, Lima 11, Perú.

\section{REFERENCIAS BIBLIOGRÁFICAS}

1. Real Academia Española. Diccionario de la lengua española. Madrid: Real Academia Española; 2001.

2. Miyahira J. Calidad en los servicios de salud: ¿Es posible? Rev Medica Hered. 2001; 12(3):75-7.

3. Parasuraman A, Zeithaml VA, Berry LL. A conceptual model of service quality and its implications for future research. J Mark. 1985;49(4):41.

4. Ladhari R. A review of twenty years of SERVQUAL research. Int J Qual Serv Sci. 2009; 1(2):172-98.

5. Aghamolaei T, Zare S. Quality gap of educational services in viewpoints of students in Hormozgan University of medical sciences. BMC Med Educ. 2008; 8(1):34.

6. Parasuraman A, Zeithaml VA, Berry LL. SERVQUAL: A multiple-item scale for measuring consumer perceptions of service quality. J Retail. 1988;64(1):12-40.
7. Reátegui LA, Izaguirre MH. Calidad de la rotación de pediatría comunitaria, desde la percepción del estudiante. An Fac Med. 2013;74(2):117-21.

8. Ajibisheh MN, Ajibisheh MN, Nejad FB. Quality enhancement in medical education. Lex Sci Intl J. 2007; 14: 212.

9. Kebriaei A, Roudbari M. Quality gap in educational services at Zahedan University of Medical Sciences: Students viewpoints about current and optimal condition. Iran J Med Educ. 2005;5(1):53-61.

10. Cieza J, O. Chincha O, Díaz E, et al. Estudio de satisfacción de los estudiantes de una Facultad de Medicina de Lima-Perú durante el año 2002: ¿Cuán satisfechos están nuestros estudiantes? Lima: Segundo Congreso Internacional de Científicos Peruanos de la Red Mundial de Científicos Peruanos.

11. Tantas GZ. Evaluación de la calidad de enseñanza desde la perspectiva de satisfacción de los estudiantes de medicina de la Universidad Nacional Federico Villarreal y Universidad Privada Ricardo Palma, Lima - Perú, 2008. Lima: Universidad Nacional Federico Villarreal; 2009.

Recibido: 01/09/2017

Aceptado: 26/12/2017 